\title{
External Validation of the 4C Mortality Score among COVID-19 Patients Visiting the Emergency Department or admitted to Hospital in Ontario, Canada
}

\author{
Aaron Jones ( $\sim$ jonesa13@mcmaster.ca ) \\ McMaster University \\ Tyler Pitre \\ McMaster University \\ Mats Junek \\ McMaster University \\ Jessica Kapralik \\ McMaster University \\ Rina Patel \\ McMaster University \\ Edward Feng \\ McMaster University \\ Laura Dawson \\ McMaster University \\ Jennifer Tsang \\ McMaster University \\ MyLinh Duong \\ McMaster University \\ Terence Ho \\ McMaster University \\ Marla Beauchamp \\ McMaster University \\ Andrew Costa \\ McMaster University \\ Rebecca Kruisselbrink \\ McMaster University
}

Research Article 
Keywords: COVID-19, Clinical risk prediction models, External validation

Posted Date: March 11th, 2021

DOI: https://doi.org/10.21203/rs.3.rs-268410/v1

License: (c) (1) This work is licensed under a Creative Commons Attribution 4.0 International License. Read Full License

Version of Record: A version of this preprint was published at Scientific Reports on September 20th, 2021. See the published version at https://doi.org/10.1038/s41598-021-97332-1. 


\section{Abstract}

Objectives: Risk prediction scores are important tools to support clinical decision-making for patients with coronavirus disease (COVID-19). The objective of this paper was to validate the $4 \mathrm{C}$ mortality score, originally developed in the United Kingdom, for a Canadian population.

Methods: We conducted an external validation study within a registry of COVID-19 positive emergency department visits and hospital admissions in the Kitchener-Waterloo and Hamilton regions of southern Ontario between March 4 and January 9, 2020. We examined the validity of the $4 \mathrm{C}$ score to prognosticate in-hospital mortality using the area under the receiver operating characteristic curve (AUC) with $95 \%$ confidence intervals calculated via bootstrapping.

Results: The study included 560 individuals, of whom 115 (20.5\%) died in-hospital. Median age was 69 years and 281 individuals (51\%) were male. The AUC of the $4 \mathrm{C}$ score was $0.83,95 \%$ confidence interval 0.79-0.87. Mortality rates across the pre-defined risk groups were 0\% (Low), 3.2\% (Intermediate), 25.9\% (High), and 59.5\% (Very High). The AUC was 0.80 (0.76-0.85) among hospital inpatients.

Interpretation: The 4C score is a valid tool to prognosticate mortality from COVID-19 in Canadian emergency departments and hospitals.

\section{Introduction}

Coronavirus disease (COVID-19) caused by the Severe Acute Respiratory Syndrome Virus 2 (SARS-CoV-2) can progress to acute respiratory distress syndrome, multiorgan failure, and death in some individuals ${ }^{1}$. The COVID-19 infection-fatality risk varies widely with age, ranging from $<0.1 \%$ for children and younger adults to greater than $10 \%$ for adults 75 and older $^{2}$. The clinical presentation and progression of COVID19 in patients is highly variable ${ }^{3}$, which makes it difficult for clinicians to triage patients and determine their risk of poor outcomes. A simple, validated prognostic tool utilizing data that is available at presentation can help clinicians better prognosticate and make clinical decisions. Numerous tools to predict mortality in COVID-19 patients have been developed, but many are limited due to small derivation cohort sizes and/or inadequate validation ${ }^{4}$.

The $4 \mathrm{C}$ mortality score is an accessible risk stratification score developed by the International Severe Acute Respiratory and Emerging Infections Consortium (ISARIC) ${ }^{5}$. It was derived and internally validated on a large, diverse cohort within the United Kingdom but needs external validity to confirm its generalizability. The objective of this study was to validate the ability of the $4 \mathrm{C}$ score to prognosticate mortality in COVID-19 patients presenting to the emergency department or admitted to hospital in Ontario, Canada.

\section{Methods}




\section{Study design and setting}

We conducted a validation study using records from the McMaster Multi-Regional Hospital Coronavirus Registry (COREG). COREG is a multi-center data registry collecting information on positive COVID-19 cases in the Kitchener-Waterloo and Hamilton regions of southern Ontario, Canada. The registry includes COVID-19-related emergency department (ED) visits and hospital admissions from six hospitals across the regions, including both academic and community centres.

\section{Participants}

We selected all patients admitted up to four weeks before time of analysis, which included admissions between March 4th, 2020 and January $7^{\text {th }}, 2020$ (n=596). We excluded patients with no clinical data collected at presentation $(n=36)$, for a final sample size of 560 .

\section{Measures}

Data in COREG were abstracted from electronic medical records using a modified case report form published by ISARIC and the World Health Organization. We utilized demographic and clinical data at presentation, typically from the emergency department. For patients who were directly admitted, we utilized the first day of inpatient records.

\section{$\underline{4 C \text { mortality score }}$}

The 4C mortality score was derived and validated within the ISARIC World Health Organization Clinical Characterisation Protocol UK study ${ }^{6}$. The score was derived from a population of over 35,000 hospital inpatients validation on over 22,000 inpatient records indicated good discriminability (area under the receiver operating characteristic curve $(\mathrm{AUC})=0.77)^{5}$.

The $4 \mathrm{C}$ score incorporates age, sex, comorbidities, respiratory rate, peripheral oxygen saturation, Glasgow Coma Scale, blood urea nitrogen, and C-reactive protein. We adapted the score to match our available data as the Glasgow Coma Scale was not collected at presentation we replaced this risk factor with the documented presence of altered consciousness or confusion (Supplementary Table S1). The 4C score ranges from 0 to 21 with risk groups defined as Low (0-3), Intermediate (4-8), High (9-14), and Very high $(\geq 15)$.

\section{Outcome}

The primary outcome was in-hospital mortality. 


\section{Statistical Analysis}

We validated the 4C score using the AUC, with 95\% confidence intervals calculated via bootstrapping with 2000 resamples. Missing data was treated using multiple imputation with chained questions.

We plotted the proportion of patients who died in hospital by 4C score and risk group and compared them to the initial derivation work in the UK. As a sensitivity analysis, we also examined the discriminability of the $4 \mathrm{C}$ for only hospital inpatients $(n=484)$. All analysis was done in $R$ 4.0.3. ${ }^{7}$

\section{Ethics approval}

Our study received ethics approval from the Tri-hospital Research Ethics Board and the Hamilton Integrated Research Ethics Board, who waived the requirement for informed consent as the data for this study was retrospectively collected from hospital medical records. All methods were performed in accordance with relevant guidelines and regulations.

\section{Results}

Our study included 560 patients, of which 484 (86\%) were admitted to hospital, and 115 (21\%) died (Table 1). Median age was 69 with patients split equally between males (51\%) and females. After imputation, the low risk group contained $13.5 \%$ of patients, the intermediate group contained $25.3 \%$, the high risk group contained $49.4 \%$ and the very high risk group contained $11.6 \%$.

\section{Validation}

The AUC of the $4 \mathrm{C}$ score was $0.83,95 \% \mathrm{Cl}(0.79-0.87)$. Mortality rates within the risk groups were $0 \%$ (Low), 3.2\% (Intermediate), 25.9\% (High), and 59.4\% (Very High) (Fig. 1). These mortality rates are slightly lower the rates reported in the original research $(1.2 \%, 9.9 \%, 31.4 \%$, and $61.5 \%)$. The performance of the model was slightly lower when only hospital inpatients were considered (AUC $=0.80,95 \% \mathrm{Cl}$ : $0.76-0.85)$. 
Table 1

Characteristics of COVID-positive hospital inpatients and emergency department patients in the Kitchener-Waterloo region of Canada, March 2020 - January 2021.

\begin{tabular}{|c|c|}
\hline \multirow[t]{2}{*}{ Characteristic } & $N(\%)^{1}$ \\
\hline & $n=560$ \\
\hline Age, yrs, (median Q1, Q3) & $69(54,82)$ \\
\hline Sex, males & $283(51)$ \\
\hline \multicolumn{2}{|l|}{ Region } \\
\hline Kitchener-Waterloo & $405(62)$ \\
\hline Hamilton & $109(27)$ \\
\hline \multicolumn{2}{|l|}{ Presenting Symptoms } \\
\hline Temperature (C), (median Q1, Q3) & $37.1(36.7-38.1)$ \\
\hline Cough & $352(63)$ \\
\hline Shortness of breath & $319(57)$ \\
\hline Fatigue & $269(48)$ \\
\hline Altered Consciousness / Confusion & $106(19)$ \\
\hline Heart Rate, per minute, (median Q1, Q3) & $91(78,104)$ \\
\hline RR rate, per minute, (median Q1, Q3) & $20(18,24)$ \\
\hline Oxygen saturation, (median Q1, Q3) & $95(93,97)$ \\
\hline \multicolumn{2}{|l|}{ Comorbidities } \\
\hline Chronic lung disease ${ }^{2}$ & $82(15)$ \\
\hline Chronic cardiac disease & $86(15)$ \\
\hline Diabetes & $178(32)$ \\
\hline Chronic liver disease & $14(3)$ \\
\hline Chronic kidney disease & $61(11)$ \\
\hline Chronic neurological disease & $65(10)$ \\
\hline Cancer & $69(12)$ \\
\hline Obesity $^{3}$ & $55(10)$ \\
\hline Rheumatologic disease & $30(5)$ \\
\hline
\end{tabular}




\begin{tabular}{|c|c|}
\hline Characteristic & $\begin{array}{l}N(\%)^{1} \\
n=560\end{array}$ \\
\hline Dementia & $81(14)$ \\
\hline HIV /AIDs & $<6$ \\
\hline Number of comorbidities, (median Q1, Q3) & $1(0,2)$ \\
\hline \multicolumn{2}{|l|}{ Biomarkers } \\
\hline Blood Urea Nitrogen, mmol/L, (median Q1, Q3) & $6.6(4.6,10.6)$ \\
\hline C-reactive protein, mg/L, (median Q1, Q3) & $66(19,147)$ \\
\hline White blood cells, $\times 10^{9} / \mathrm{L},($ median Q1, Q3) & $6.6(5.1,9.5)$ \\
\hline Platelets, x109/L, (median Q1, Q3) Q3) & $211(162,282)$ \\
\hline Neutrophils, $\times 10^{9} / \mathrm{L}$, (median Q1, Q3) & $5.0(3.4,7.3)$ \\
\hline Lymphocytes, $x 10^{9} / \mathrm{L}$, (median Q1, Q3) & $1.0(0.7,1.4)$ \\
\hline \multicolumn{2}{|c|}{ 1. Percentages are denominated by number of non-missing cases } \\
\hline \multicolumn{2}{|l|}{ 2. Does not include asthma } \\
\hline 3. As defined by staff & \\
\hline
\end{tabular}

\section{Interpretation}

We found that the $4 \mathrm{C}$ mortality score is a valid tool to prognosticate mortality among COVID-19 patients presenting to the emergency department or upon admission to hospitals in a Canadian population. We observed an AUC of 0.83 in our data, which is higher than was reported in the original derivation research in the United Kingdom but similar to validation conducted in Europe ${ }^{8}$.

Clinical risk prediction models are critical tools that can be used to identify and prioritize high-risk patients. The proliferation of COVID-19 risk models is evidence of the demand for an accurate, accessible, and generalizable tool ${ }^{9}$. Our finding that the $4 \mathrm{C}$ mortality score is valid in a jurisdiction distinct from the location of the original derivation and subsequent validation provides evidence for the generalized prognostic ability of the score.

We have demonstrated that it is an informative clinical tool within academic and community hospital care settings in Ontario, which are similar to care settings across Canada. 
The AUC we observed was higher than in the original research, which is unusual for external validation, but this was also true in other validation studies done in the United Kingdom and the Netherlands 8,10 . The higher discriminability in our study may in part because we examined a more heterogeneous cohort that included patients who visited the emergency department but were not admitted to hospital. The 4C score was likely able to identify that these patients were at low risk and assign them lower scores. Overall, the AUC was only slightly lower in the sensitivity analysis that only examined hospital inpatients and inclusion of outpatients into the validation population adds further value to the study.

\section{Limitations}

A key limitation of our study is that we were only able to include data from two regions within southern Ontario. While similarities between health systems across Canada suggest our findings will have excellent generalizability to other Canadian provinces and territories, our results may not generalize to geographically remote settings or to jurisdictions with substantially different health systems.

\section{Conclusion}

The $4 \mathrm{C}$ mortality score is an adaptable, valid prognostic tool for use in Canadian emergency departments and hospitals. It can be used to identify and prioritize care for COVID-19 patients at high risk of death.

\section{Declarations}

\section{Funding Statement}

This study leveraged data from the COREG registry, which is supported by a grant from the Canadian Institutes of Health Research (CIHR) (172754) and from the Hamilton Academic Health Sciences Organization (HAHSO) (HAH-21-04).

\section{Data Sharing Statement}

The data used in this study can be accessed for research purposes by submitting a request through the COREG data access portal. (https://www.coregontario.ca/info-data-access)

\section{Code availability.}

Code is available upon request

\section{Conflicts of interest}

None 


\section{Ethics approval}

This study received ethics approval from the Tri-hospital Research Ethics Board and the Hamilton Integrated Research Ethics Board.

\section{Contributor's statement}

Study conception and design: AJ, TP, MD, TH, MB, AC, RK

Acquisition of data: TP, MJ, JK, RP, EF, LD

Analysis and Interpretation: AJ, TP, MJ, JK, JT, MD, MB

AJ wrote the initial draft of the manuscript and all authors critically revised it for important intellectual content. All authors agree to act as guarantors of the work.

\section{Acknowledgements}

This study leveraged the McMaster Coronavirus (COVID-19) Registry (COREG) led by Drs. Andrew Costa (NPI), Marla Beauchamp (co-PI), MyLinh Duong (co-PI), Rebecca Kruisselbrink (co-PI), Terence Ho (co-PI), and Jennifer LY Tsang (co-PI) and included the following institutions: Grand River Hospital, St. Mary's General Hospital, Hamilton Health Sciences, St. Joseph's Healthcare Hamilton, and the Niagara Health System. The COREG acknowledges the following individuals for their contributions: Muneeb Ahmed, Darly Dash, Megan Donaghy-Hughes, Hannah Farnworth, Stefan Jevtic, Catherine Lee, Candice Luo, Sarah MacGregor, Michael Mallender, Adhora Mir, Vivek Patel, Nivedh Patro, Noam Raiter, Pranali Raval, Brittany Salter, Adib Shamsuddin, Laura Spatafora, Xinxin Tang, Cooper Webb, Kristin Wright, Grace Xu, and Zaka Ahmad Zia.

\section{References}

1. Wu, C. et al. Risk Factors Associated With Acute Respiratory Distress Syndrome and Death in Patients With Coronavirus Disease 2019 Pneumonia in Wuhan, China. JAMA Intern. Med. 180, 934943 (2020).

2. Yang, W. et al. Estimating the infection-fatality risk of SARS-CoV-2 in New York City during the spring 2020 pandemic wave: a model-based analysis. Lancet Infect. Dis. 0, (2020).

3. Struyf, T. et al. Signs and symptoms to determine if a patient presenting in primary care or hospital outpatient settings has COVID-19 disease. Cochrane Database Syst. Rev. (2020) doi:10.1002/14651858.CD013665.

4. Wynants, L. et al. Prediction models for diagnosis and prognosis of covid-19: systematic review and critical appraisal. BMJ 369, (2020). 
5. Knight, S. R. et al. Risk stratification of patients admitted to hospital with covid-19 using the ISARIC WHO Clinical Characterisation Protocol: development and validation of the 4C Mortality Score. BMJ 370, (2020).

6. Docherty, A. B. et al. Features of 20133 UK patients in hospital with covid-19 using the ISARIC WHO Clinical Characterisation Protocol: prospective observational cohort study. BMJ 369, (2020).

7. R Core Team. R: A language and environment for statistical computing. (2020).

8. Wellbelove, Z., Walsh, C., Perinpanathan, T., Lillie, P. \& Barlow, G. Comparing the 4C mortality score for COVID-19 to established scores (CURB65, CRB65, qSOFA, NEWS) for respiratory infection patients. J. Infect. (2020) doi:10.1016/j.jinf.2020.10.015.

9. Gupta, R. K. et al. Systematic evaluation and external validation of 22 prognostic models among hospitalised adults with COVID-19: An observational cohort study. Eur. Respir. J. (2020) doi:10.1183/13993003.03498-2020.

10. Dam, P. M. E. L. van et al. Performance of prediction models for short term outcome in COVID-19 patients in the emergency department: a retrospective study. medRxiv 2020.11.25.20238527 (2020) doi:10.1101/2020.11.25.20238527.

\section{Figures}

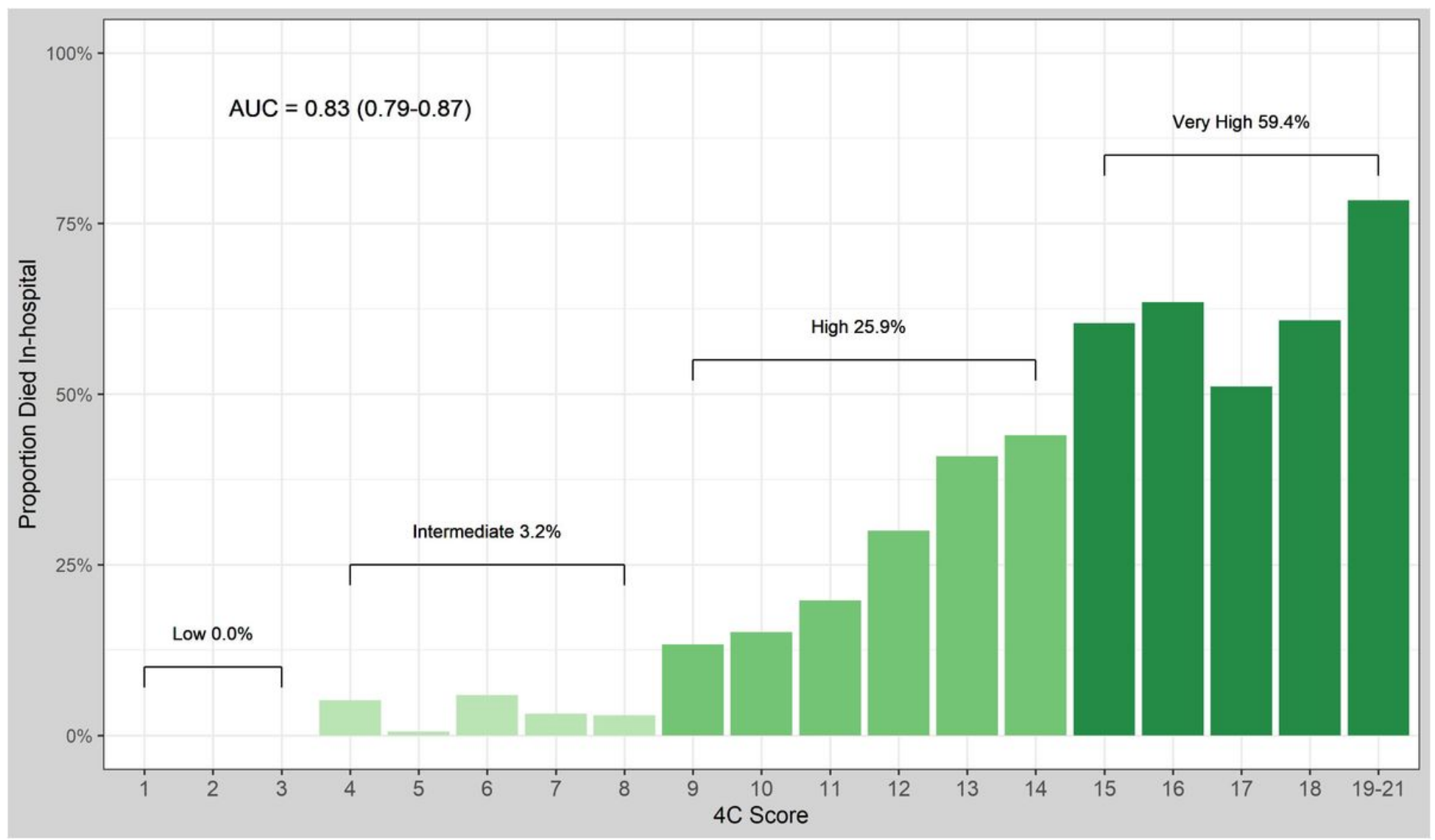

Figure 1 
Performance of the $4 \mathrm{C}$ mortality score to predict in-hospital mortality among COVID-19 patients

\section{Supplementary Files}

This is a list of supplementary files associated with this preprint. Click to download.

- SupplementaryTableS1.docx 